Association for Information Systems AIS Electronic Library (AISeL)

GlobDev 2008

Proceedings Annual Workshop of the AIS Special Interest Group for ICT in Global Development

Winter 12-13-2008

\title{
E-Health Readiness Framework from Electronic Health Records Perspective
}

JunHua Li

The University of New South Wales

Lesley Pek Wee Land

The University of New South Wales

Subhagata Chattopadhyay

The University of New South Wales

Pradeep Ray

The University of New South Wales

Follow this and additional works at: http://aisel.aisnet.org/globdev2008

\section{Recommended Citation}

Li, JunHua; Land, Lesley Pek Wee; Chattopadhyay, Subhagata; and Ray, Pradeep, "E-Health Readiness Framework from Electronic Health Records Perspective" (2008). GlobDev 2008. 4.

http://aisel.aisnet.org/globdev2008/4 


\title{
E-HEALTH READINESS FRAMEWORK FROM ELECTRONIC HEALTH RECORDS PERSPECTIVE
}

\author{
JunHua Li $\mathrm{Li}^{\mathrm{a}, \mathrm{b}}$ \\ Lesley Pek Wee Land ${ }^{\mathrm{a}, \mathrm{b}}$ \\ Subhagata Chattopadhyay ${ }^{\mathrm{a}, \mathrm{b}}$ \\ Pradeep Ray ${ }^{\mathrm{a}, \mathrm{b}}$ \\ The University of New South Wales \\ ${ }^{a}$ The School of Information Systems, Technology and Management \\ ${ }^{\mathrm{b}}$ Asia Pacific u-Health research Centre (APuHC) \\ Sydney NSW 2052, Australia.
}

\begin{abstract}
E-Health systems include applications of information communication technologies to promote healthcare services support, delivery and education. The success of an E-Health system is very much dependent on the success of EHR systems, as EHR forms the core of any E-Health system. Readiness assessment has been identified as an essential requirement for the success of EHR in terms of adoption rate and/or acceptance. Through a literature review of current E-Health readiness frameworks, it is observed that most studied components reflect healthcare providers' and organisational perspectives but there is an inconsistent coverage of the evaluation components. Further, an unclear measure of readiness levels poses another problem for E-Health readiness assessment. This paper presents an E-Health readiness assessment framework by integrating components of each reviewed framework and quantifying constructs (a graph-based approach) within the new framework.
\end{abstract}

Keywords: E-Health, Electronic Health Records (EHR), Readiness, Assessment, Evaluation, Constructs, Quantification, Graph-based 


\section{INTRODUCTION}

To promote development for developing countries, healthcare issues must be tackled first. Healthcare, which spans across all ages, genders, races, culture and geographical boundaries, is an area of concern to all population. Many countries (especially developing countries) are plagued with critical healthcare issues such as chronic, infectious and/or pandemic diseases, a lack of basic healthcare programmes and facilities and a shortage of skilled healthcare workers (WHO, 2006; Watts et al. 2005). Poor healthcare obstructs prosperity and business profitability, and does not help reduce poverty. More importantly, it directly affects the mortality levels of the people living in these countries. Therefore, proper management of healthcare is a prime concern for the development of developing countries.

Electronic Health (E-Health) facilitates healthcare to reach remote populationhitherto nor properly serviced by traditional means. However, readiness is very important to all aspects of E-Business in developing countries (e.g. E-Gov, E-Finance) (Heeks, 2008) as recognised by the UN and WHO through the Declarations in last World Health Assembly and the World Sysmposium on Information Society (WSIS) attended by ministers in all countries.

Healthcare services are increasingly needed by people and should be efficiently provided and made fully accessible to all (Haglund, 2002). E-Health has been defined as an amalgamation of healthcare system and Information Communication Technology (ICT) to enable better health and healthcare (Nykanen, 2006). According to Silber (2003), E-Health is an "application of information and communication technologies (ICT) across the whole range of functions that affect health”. It is also an emerging field in the intersection of medical informatics, public health and business, and refers to health services and information delivered or enhanced through the Internet and related technologies (Eysenbach, 2001). E-Health kicks off a revolution to traditionally paper-based health and healthcare. As one of the main sponsors of this 
revolution, World Health Organisation (WHO) (2005) claimed E-Health applications as the use of digital data transmitted, sorted and retrieved electronically - in support of healthcare, both at the local site and at a distance. The broader sense of E-Health is its ability to directly support prevention, patient diagnosis and patient management, and care. There is therefore a consensus that E-Health includes applications of ICT to promote healthcare services support, delivery and education.

Regarding the objective of E-Health systems, it is different from that of other information systems, e.g. ERP and CRM. ERP and CRM systems aim to improve effectiveness and efficiency of business processes for productivity and profitability whereas improving well-being and health of people (i.e. customer value) are the key objective for E-Health systems.

Electronic Health Records (EHR) (consisting of three components, which have been identified and developed by Dicksinson et al. (2004): Direct care EHR functions, Supportive EHR requirements, and EHR Information Infrastructure.) forms the core of any E-Health system. Hence the success of an E-Health system is very much dependent on the success of the EHR systems. Information systems (IS) researchers have recognised the problem of sustainability and complexity in health information systems implementations especially in developing countries (e.g. (Braa et al. 2004; Miscione, 2007)). This is evidenced by the encouragement to undertake high-visibility and high impact research that takes a greater macro focus when examining the transformational nature of IT (Agarwal \& Lucas, 2005; Walsham et al. 2007). In the healthcare area, this means that researchers should take a more holistic view of the environment in which the health information systems are implemented and deployed. Accordingly, it is important to solicit data from diverse stakeholders at different levels of healthcare and related organisations who have (in)direct interest in such projects (e.g. distributed researchers, potential end users, technical developers, programme sponsors). Evaluation research by studying the context of healthcare information systems implementation fits in with this recommendation. 
Evaluation undertaken before E-Health system development (i.e. pre-implementation) is particularly pertinent due to its complexity (BC eHealth Steering Committee, 2005), usual to any IS project. As with the implementation of any information system in an organisational context, the acceptance of any information system requires proper planning and management for change (Callioni, 2006). With EHR implementations, change occurs not simply due to the introduction of ICT infrastructure but also because the job design of interconnected health professionals should be reengineered to effectively and efficiently accommodate the technology (Eric et al., 2006). EHR implementations represent a disruptive change in the healthcare workplace. E-Health readiness assessment, a part of pre-implementation evaluation, becomes an essential requirement prior to implementation (Jennett et al. 2003; Demiris et al. 2004).

The key objectives of this paper are to:

- Identify key components for E-Health readiness assessment;

- Design an integrated E-Health readiness framework and

- Introduce a conceptual method to quantify constructs within the framework in order to reveal E-Health readiness status.

The layout of the paper is as follows. Section 2 discusses the background information and motivation to study E-Health readiness; then reviews current E-Health readiness frameworks. Section 3 identifies the existing research gaps. Section 4 presents an integrated E-Health readiness framework (conceptual) with quantified constructs using a graph-based approach. We conclude in Section 5 with a summary of our study, some tentative implications, current limitations and future scope of E-Health research.

\section{BACKGROUND AND MOTIVATION}

Evaluation has been defined as an act of measuring or exploring properties of an IS (in planning, development, implementation, or operation phase), the result of which informs a decision to be made concerning that system in a specific context 
(Ammenwerth et al. 2004). This definition considers evaluations that happen at different phases of an IS lifecycle and thus help to achieve sustainability in its implementations. In brief, evaluation is a broad term for various methods and strategies for identifying the effects and assessing the value, feasibility, or other qualities of a technology, programme, or policy (Field, 1996).

Most evaluation studies conducted in the healthcare context take place in the post-implementation phase, which is, after an E-Health system is delivered (e.g. Ammenwerth et al. 2001; Overhage et al. 2001). While post-implementation evaluations are crucial for assessing the merit, success and value of systems, contributing to evidence-based practices, and 'learning from experience' (Alexander, 2007), pre-implementation evaluations aim to give direction for decision making with regard to subsequent development or implementation tasks (Brender, 2006). During this analysis and the planning stage, the evaluation for E-Health systems covers (Brender, 2006):

- Relevance: assessing whether the solution is entirely able to solve the current problems and meet the demands and requirements of the organisation;

- Problem Areas: identifying the weakness and the elements of risk in the solution;

- Feasibility: assessing the organisational resources needed to implement the chosen solution;

- Completeness and Consistency: assessing whether the solution is a coherent entity that is neither over nor undersized; and

- Elements of Risk: assessing whether there are any external conditions outside organisational control that will involve substantial risk to the project.

Accordingly, numerous methods can be used, e.g. balanced scorecard (Gordon et al. 1999; Protti, 2002), field study (Brender, 1999), focus group interview (Steward et al. 1990), organisational readiness (Khoja et al. 2007) and the like (Brender, 2006). E-Health readiness assessment, for example, is a way of identifying the potential causes of failure to innovate. A lack of readiness shows organisational inability to 
undergo transformation during the implementation of EHR (Brender, 2006), leading to sheer failure at the end. Failure occurs when the system is completely abandoned. In many other instances, system may not exhibit complete failure straightaway, but show signs of system abandonment and user dissatisfaction through lack of active use and lack of management commitment to system maintenance. These intermediate stages are indications of a lack of readiness in the system implementation.

With the introduction of EHR systems, healthcare can be improved by providing better evidence-based healthcare (Overhage et al. 2001) (e.g. higher levels of completeness, accuracy, validity, and utility of patient health records for clinical diagnoses (Hassey et al. 2001; Embi et al. 2004; Sek et al. 2007)), providing better clinical decision support (e.g. Stewart et al. 2007; Protti, 2007) and electronic prescribing (Schade et al. 2006), and increasing medical practice efficiency (Ammenwerth et al. 2004). Although interest in automating the health records is generally high, the literature however informs us that EHR systems do not always succeed in terms of adoption rate and/or acceptance. The adoption of EHR systems still tremendously differs from one to another. Some developed countries like Sweden, The Netherlands, and Australia have more than half of their primary care physicians using EHR, respectively 90\% in Sweden, 62\% in Denmark and 55\% in Australia (Taylor et al. 2002; Terry, 2004). In other developed countries, when information technology was also available (Watson, 2006), adoption nonetheless appeared to be significantly lower, only $1.2 \%$ of all hospitals and $2.6 \%$ of all clinics adopted EHR in Japan (MHLW, 2002) and less than 18\% of physicians used EHR in their offices in the US (Burt \& Hing, 2005). The key obstacle of low EHR adoption was certainly not the availability of information technology alone, but more likely other factors such as the availability of technical support (Watson, 2006). This latter factor is exacerbated when the use of electronic systems is out of reach to many healthcare practitioners such as poor ICT experience of healthcare providers and administrators (Watson, 2006). 
While EHR adoption has been low in some developed countries, the success of EHR adoption has also been low for developing countries, which are generally resource constrained (e.g. insufficient E-Health infrastructure), due to lack of (i) required ICT support, (ii) awareness among stake holders, and (iii) a drive to adopt new system or functions (Watts et al. 2005; Diero et al. 2006). Moreover, costs, available information technology infrastructure (including a lack of data processing facilities), and a lack of technical expertise and computer skills of staff are major issues which would need to be addressed before EHR implementation is possible (Watson, 2006). In other words, readiness assessment for the innovation becomes an essential requirement for the successful implementation and use of EHR (and hence E-Health). This is because sufficient preparation and pre-planning from an organisational perspective enable customisation of the system and ensure minimal workflow disruptions during and after implementation (Fullerton et al. 2006).

\section{REVIEW OF CURRENT E-HEALTH READINESS}

\section{FRAMEWORKS}

In order to investigate multiple healthcare providers' view (e.g. physicians, nurses and administrative personnel) for the readiness evaluation of E-Health applications, Campbell et al. (2001) developed a readiness framework by conducting semi-structured interviews (regarding both the video and computer components of telemedicine), followed by thematic analysis. Results of thematic analysis reveal six themes (Campbell et al. 2001):

- Turf: a threat to healthcare providers' livelihood or professional autonomy or both;

- Efficacy: desire to know that E-Health applications will fill a functional need in healthcare providers' practice before they invest time and money in making such a big change;

- Practice context: barriers to adopting E-Health applications;

- Apprehension: as a human aversion to change; 
- Time to learn: hesitancy among the providers to take the time to learn a new technology and to persuade patients of its worth;

- Ownership: participants who were professionally and emotionally invested in the technology - stakeholders who acknowledged its benefits, adapted it to their needs, and tried to help others learn.

These six themes comprise the framework to understand three categorised organisational settings, i.e., "fertile soil, somewhat fertile soil, and barren soil" (Campbell et al. 2001). Change strategies are also suggested to every readiness setting. Campbell et al. provided a mechanism for determining and then dealing with three different levels of readiness for implementing E-Health applications. Nevertheless, the mechanism does not involve organisational, public or patient readiness for E-Health (only from healthcare providers' view). Furthermore, Campbell et al.'s framework (2001) has not been tested.

Another readiness evaluation study followed the philosophy of existing readiness scales (the Organisational Information Technology/Systems Innovation Readiness Scale (OITIRS) and the organisational and functioning readiness for change (ORC) scale); the evaluation is also from the healthcare providers' viewpoint. This framework involves staff profiles, staff exposure to technology and institutional resources (Demiris et al. 2004). Using previously validated instruments, it captures organisational readiness for E-Health. Demiris et al.’s framework, however, seems to focus solely on assessing practitioner readiness instead of organisational readiness, as instruments primarily includes staff profiles and staff exposure to technology.

By contrast, the readiness framework from Jennett et al. (2003; 2004) is relatively comprehensive in terms of the evaluation scope. Sixteen semi-structured telephone interviews to four sets of stakeholders (patient, practitioner, organisation and public) (Project report for CANARIE, 2002) were conducted to examine complex social, political, organisational and infrastructure factors. As a result, four types of readiness 
were found:

- Core readiness refers to the realisation of needs and expressed satisfaction with the present situation and conditions;

- Engagement readiness involves the active participation of people in the idea of E-Health. In this process, people weigh the advantages and disadvantages of E-Health, assess risk, and question E-Health as a solution;

- Structural readiness focuses on the establishment of efficient structures as a foundation for successful E-Health projects within an organisation for example, human, technical, training, policy and funding;

- Concern of non-readiness is expressed as a perceived lack of need or a failure to recognize a need for change and implementation of E-Health technology. (Jennett et al. 2005)

Six common factors mentioned below were identified within each type of the readiness (Jennett et al. 2005):

- Core readiness refers to recognised need for the service by evaluators, along with an expressed dissatisfaction with existing service or circumstance;

- Structural readiness is concerned with whether an organisation includes adequate human resources, training, policies, funding and appropriate equipment that functioned properly or was easily repaired;

- Projection of benefits means the benefits E-Health could bring, such as reduce the need to travel and improve access to service;

- Assessment of risk involves healthcare practitioners' demands on working time and professional liability to decide whether to trust the information available to them through web-based applications for practitioners; privacy and the obtaining of reliable information for patients; a fear that E-Health services would replace the existing healthcare system is reflected for the public perspective; a financial risk especially in a short term is presented for organisations;

- Practitioners' awareness and education refer to understanding the various applications, their potential benefits and limitations; 
- Intra-group and inter-group dynamics means communication and cooperation within or across the communities of interest.

Jennett et al.'s framework suggests a method to determine overall readiness categorisation. It stresses the importance of end-users' ownership of innovation adoption by assessing organisational, health provider, public and patient readiness for E-Health. However, tool reliability has not been assessed and the study provides little information regarding demographics or current technological practices.

Another proposed framework by Wickramasinghe et al. (2005) is concerned with three domains relevant to E-Health readiness - practitioner, organisation and public; it highlights the key elements that are required for successful E-Health initiatives. Wickramasinghe et al.'s framework provides a tool that allows analysis beyond the quantifiable data into a systematic synthesis of the major four impacts and four pre-requisites, and the implications of these pre-requisites and impacts to the goals of E-Health, such as efficiency, evidence-based and preventive medicine. (Figure 1)

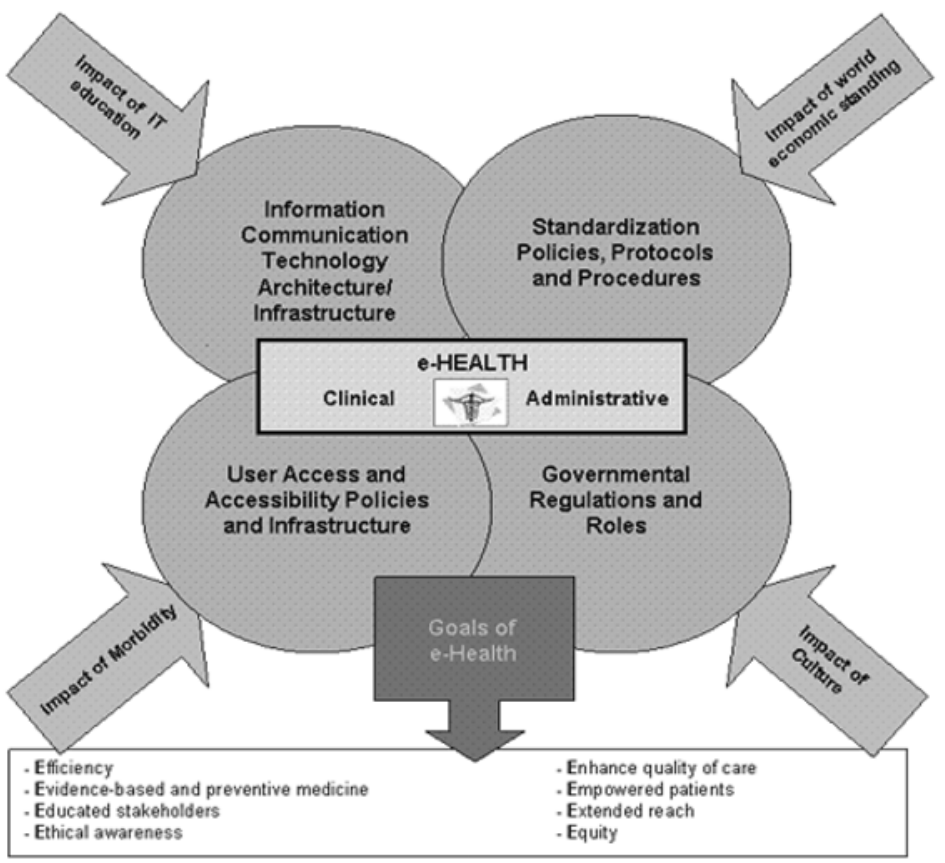

Figure 1. E-health Readiness Framework, Wickramasinghe et al. (2005) 
Wickramasinghe et al.’s framework contains four main prerequisites:

- Information communication technology (ICT) architecture/ infrastructure: a sound technical infrastructure (phone lines, fibre trunks and submarine cables, telecommunications, electricity, access to computers etc) is an essential ingredient to the undertaking of E-Health initiatives by any nation;

- Standardisation policies, protocols and procedures: E-Health by definition spans many parties and geographical dimensions. To enable such far reaching coverage, a significant amount of document exchange and information flow must be accommodated. Standardisation is the key to this, using widely and universally accepted protocols such as TCP/IP and http;

- User access and accessibility policies and infrastructure: access to e-commerce is defined by the WTO (World Trade Organisation) as consisting of two critical components: access to Internet services and access to e-services (Panagariya, 2000). The former deals with the user infrastructure whereas the latter pertains to specific commitments to electronically accessible services;

- Governmental regulation and control: the key challenges regarding E-Health use include: cost effectiveness; i.e., less costly than traditional healthcare delivery; functionality and ease of use, i.e., they should enable and facilitate many uses for physicians and other healthcare users by combining various types and forms of data as well as being easy and secure to use.

Four impacts of E-Health are embedded in Wickramasinghe et al.’s framework:

- Impact of IT education: an educated population boosts the E-Health initiative;

- Impact of world economic standing: awareness of importance and critical role of Internet in a country's economy;

- Impact of morbidity rate: education/awareness and overall health standing of a country;

- Impact of cultural/social dimensions: culture, traditions and the like. 
Wickramasinghe et al.'s framework based on multiple perspectives, including organisational (e.g. ICT infrastructure), practitioner (e.g. user access) and public (e.g. governmental regulation) can be used to assess the potential of a country and its readiness for E-Health as well as its ability to maximise the goals of E-Health.

The study of Overhage et al. (2005) involves system readiness evaluation other than practitioner and organisational readiness by analysing secondary data that communities submitted for funding better healthcare programmes. Descriptive statistics and subjective evaluation were used to explore seven dimensions that an expert review panel had judged to be important determinants of a community's success in creating a health information exchange, i.e., clinical component, demonstration of community commitment and leadership, matching funds, overall technical readiness, plans for sustainable business model, use of data standards, use of replicable and scalable tools. Nevertheless, the objective of Overhage et al.'s study was not explicitly stated; it seems to be funding allocation rather than readiness evaluation because the data were the basis for an invitation to submit a proposal for the funding. Tool reliability or validity was not assessed. Furthermore, a scoring mechanism was not provided to determine readiness.

Although reviewed frameworks were not tested (Campell, 2001; Jennett 2003; 2004; Overhage, 2005), a recent study by Khoja et al. (2007) aimed to test the reliability of E-Health readiness evaluation tools for both Managers and healthcare providers with four categories of measurements in developing countries. Separate scores (Cronbach's alpha) were measured for each of the four categories in both the tools. (Table 1) Scores of core-readiness, learning readiness/technological readiness, societal readiness and policy readiness for both tools were all observed higher than 0.80 and show high reliability. 


\begin{tabular}{|c|c|c|c|}
\hline \multirow{2}{*}{\multicolumn{2}{|c|}{ Category }} & \multicolumn{2}{|c|}{ Cronbach’s Alpha Score } \\
\hline & & For Managers & For Healthcare Providers \\
\hline \multicolumn{2}{|l|}{ Overall } & 0.94 & 0.91 \\
\hline $\begin{array}{l}\text { Core } \\
\text { readiness }\end{array}$ & $\begin{array}{l}\text { i) needs-assessment and dissatisfaction with status quo; ii) } \\
\text { awareness about E-Health in the organisation; iii) comfort with } \\
\text { the use of technology; iv) trust in technology; v) planning of } \\
\text { E-Health projects; vi) overall willingness and satisfaction; and } \\
\text { vii) integration of technology; }\end{array}$ & 0.92 & 0.86 \\
\hline $\begin{array}{l}\text { Learning } \\
\text { readiness }\end{array}$ & $\begin{array}{l}\text { i) use of ICT in enhancing education for healthcare providers; } \\
\text { and ii) involvement of healthcare providers in E-Health } \\
\text { projects; }\end{array}$ & & 0.88 \\
\hline $\begin{array}{l}\text { Technological } \\
\text { readiness }\end{array}$ & $\begin{array}{l}\text { i) speed and quality of ICT; ii) availability of service and } \\
\text { support; iii) availability and affordability of hardware and } \\
\text { software; and iv) training in ICT; }\end{array}$ & 0.86 & \\
\hline $\begin{array}{l}\text { Societal } \\
\text { readiness }\end{array}$ & $\begin{array}{l}\text { i) communication with other organisations and communities; } \\
\text { ii) sharing of locally relevant content; iii) provision of care in } \\
\text { collaboration with other institutions; iv) consideration of } \\
\text { socio-cultural factors among staff; and v) consideration of } \\
\text { sociocultural factors among clients; }\end{array}$ & 0.91 & 0.81 \\
\hline $\begin{array}{l}\text { Policy } \\
\text { readiness }\end{array}$ & $\begin{array}{l}\text { i) ICT related regulations; ii) policies regarding licensure, } \\
\text { liability and reimbursement; iii) awareness and support for ICT } \\
\text { among politicians; and iv) awareness and support for ICT } \\
\text { among institutional policymakers. }\end{array}$ & 0.89 & 0.92 \\
\hline
\end{tabular}

Table 1. Cronbach's Alpha Score, Khoja et al. 2007

Each of the items within the respective four categories for managers or healthcare providers showed Pearson's correlation coefficient greater than $0.35(\mathrm{p}<0.05)$, so all the items in these categories relate appropriately with other items in the same category (Khoja et al. 2007). Although Khoja et al.'s framework can guide users to take appropriate measures and may also be used to quantitatively assess and improve E-Health readiness, the idea of E-Health is relatively new to healthcare centres in developing countries and thus it would be hard to adopt all the suggested measures to assess all levels of service.

Evaluators - and decision makers - must accept that E-Health evaluation may serve different purposes for different stakeholders, and therefore concede that no single evaluation framework or methodology is totally objective (Gagnon et al. 2005). The reviewed frameworks above, for example, were derived from different perspectives to 
evaluate E-Health readiness. (Table 2) Most studied components within the frameworks reflect healthcare providers' and organisational perspectives, however these components are different more or less from one framework to another. In terms of the readiness components from organisational perspective, ICT architecture/ infrastructure was highlighted but core readiness, identified from Jennett et al.'s framework (2003, 2004, 2005), was neglected in Wickramasinghe et al.'s (2005). This is why E-Health evaluation is often criticised for the poor quality of research design, the lack of common outcome indicators and the absence of an agreed theory (Gagnon et al. 2005). Further, how the authors of the reviewed frameworks measure or quantify readiness level is not clear even though some of the frameworks have described the readiness levels as low, medium or high level (e.g. Wickramasinghe et al. (2005)).

\begin{tabular}{|l|l|l|l|l|l|}
\hline Author and date & Patient & Provider & System & Organisational & Public \\
\hline Campbell et al. 2001 & & $\sqrt{ }$ & & & \\
\hline Demiris et al. 2004 & & $\sqrt{ }$ & & & \\
\hline Jennett et al. 2003, 2004, 2005 & $\sqrt{ }$ & $\sqrt{ }$ & & $\sqrt{ }$ & $\sqrt{ }$ \\
\hline Overhage et al. 2005 & & $\sqrt{ }$ & $\sqrt{ }$ & $\sqrt{ }$ & \\
\hline Wickramasinghe et al. 2005 & & $\sqrt{ }$ & & $\sqrt{ }$ \\
\hline Khoja et al. 2007 & & $\sqrt{ }$ & & $\sqrt{ }$ & \\
\hline
\end{tabular}

Table 2. Different perspectives of E-Health readiness framework

\section{E-HEALTH READINESS ASSESSMENT FRAMEWORK}

\section{(EHRAF)}

Existing E-Health readiness frameworks (e.g. Khoja et al. 2007) were criticised in Section 3 for their inconsistent coverage of evaluation components from healthcare providers' and organisational perspectives, and an unclear measure of readiness levels. Accordingly, an E-Health readiness assessment framework will be developed by integrating the studied components of each reviewed framework and quantifying constructs within the new framework. The steps towards the development of such a framework are as follows 
- Step 1: Identification of the components and indicators for E-health readiness assessment from healthcare providers’ and organisational perspectives;

- Step 2: Development of EHRAF using these components and indicators; and

- Step 3: Method of quantifying each of the constructs using connected graph theory.

\subsection{Identification of the Components for E-Health Readiness Assessment}

An overall E-Health readiness can be assessed from four components: Core, Engagement, Technological and Societal. These components are drawn on from the frameworks discussed in Section 3.

Core readiness refers to evaluators' realisation of problems in documentation of clinical information and healthcare providers' satisfaction with paper health records. Provision of care requires the documentation of clinical information as an intrinsic aspect of routine clinical activity and is essential from both professional and legal standpoints (Allan et al. 2000). Accordingly, core readiness assessment is concerned about patient records generation, storage and retrieval with paper-based health record systems. In particular, it involves documentation efficiency of patient records (Warshawsky et al. 1994; Allan et al. 2000), patient privacy (Gritzalis et al. 2004; Blobel, 2004) and the degree of physicians' satisfaction with completeness and accuracy of paper-based health records (PHR) (Staroselsky et al., 2006) and with sharing of patient records (Bakker, 2007). When the realised problems are more serious and dissatisfaction expressed by physicians is higher, the healthcare organisations and providers are more ready to adopt new practices (EHR) to create change (Jennett et al. 2002; Jennett et al. 2005); and vice versa.

Engagement is healthcare providers' exposure to EHR systems and willingness to accept EHR training (Campbell et al. 2001). Their exposure to EHR systems includes

- Recognition of benefits (Campbell et al. 2001; Demiris et al. 2004; Jennett et al. 2003, 2005), i.e. efficient documentation of patient records (Protti, 2007; Schade 
et al. 2006), protection of patients' privacy (Coiera et al., 2004; Galpottage et al., 2005), better provision of patients' information (Embi et al. 2004; Sek et al. 2007) and provision and sharing of timely information (Bakker, 2007; Blobel, 2006).

- Potentially negative impacts (Demiris et al. 2004; Khoja et al. 2007), i.e. high investment and low reimbursement (Leung et al. 2003; Bates, 2005), individual limitation of IT knowledge (Watson, 2006), time cost (Leung et al. 2003), worries about changes in workflow (Ash et al. 2005; Ash et al. 2007) and the management which eliminates traditional information and communication routines (Obstfelder et al. 2006).

If healthcare providers over express their fear or concern about potentially negative impacts, but have not recognised benefits of EHR and are not willing to accept EHR training, the engagement readiness is low. In contrast, high readiness is for the organisations, where healthcare providers do not over express their fear or concern about potentially negative impacts, and they have recognised the benefits of EHR and are willing to accept EHR training.

Technological readiness (e.g. Khoja et al. 2007) aims to determine the existing ICT infrastructure (hardware required for EHR applications and network) (Halamka et al. 2006), other available electronic resources (e-resources) (EHR related software), IT support personnel (Fullerton et al. 2006; Leung et al. 2003) as well as healthcare providers' past IT experience (Halamka et al. 2006). If a healthcare organisation possesses all the items enumerated below, the technological readiness is high.

- Hardware required for EHR applications: (1). Laptop; (2). Desktop; (3). Monitor; (4). Printer; (5). Document scanner; (6). Photocopier; (7). Phone; (8). TV based conferencing; (9). PC based conferencing; (10). Web cam connected; (11).

Digitalised X-Ray Equipment; (12). High resolution digital cam monitored on a Microscope

- Network: Internet access 
- EHR related software: (1). Maintenance of EHR; (2). Electronic Healthcare training; (3). To send emails; (4). Standard software package (i.e., Anti-Virus and Operation System)

- IT support personnel: (1). Provision of technical support; (2). Technical support personnel

- Healthcare providers’ past IT experience: (1). Frequency of using PC; (2).

Frequency of using e-media (e.g. emails and Internet); (3). Computer users for E-Health; (4). Training or direct experience in using EHR

Societal readiness assessment aims to understand communication links of healthcare organisations with other institutions (i.e. hospitals and administrative centres), and provision of care in collaboration with other healthcare organisations (e.g. connected diagnostic facilities like pathology/radiology) (Khoja et al. 2007). Further, it involves internal communication among healthcare providers: communication mediums and frequency (Khoja et al. 2007).

If a healthcare organisation where healthcare providers use multiple mediums to communicate with one another and the communication frequency is high, also has communication links to both hospitals and administrative centres, and provides care in collaboration with other healthcare organisations, societal readiness is high; and vice versa.

\subsection{Development of EHRAF}

E-Health Readiness Assessment Framework (EHRAF) makes an assumption that a typical EHR system (Dickinson et al. 2004) will be fully implemented. Evaluators can directly use this framework for organisations that plan to or will implement EHR systems. It includes four main readiness components: core (e.g. Jennett et al. 2003, 2004), engagement (e.g. Campbell et al. 2001), technological (e.g. Demiris et al. 2004) and societal readiness (e.g. Khoja et al. 2007). (Figure 2) 


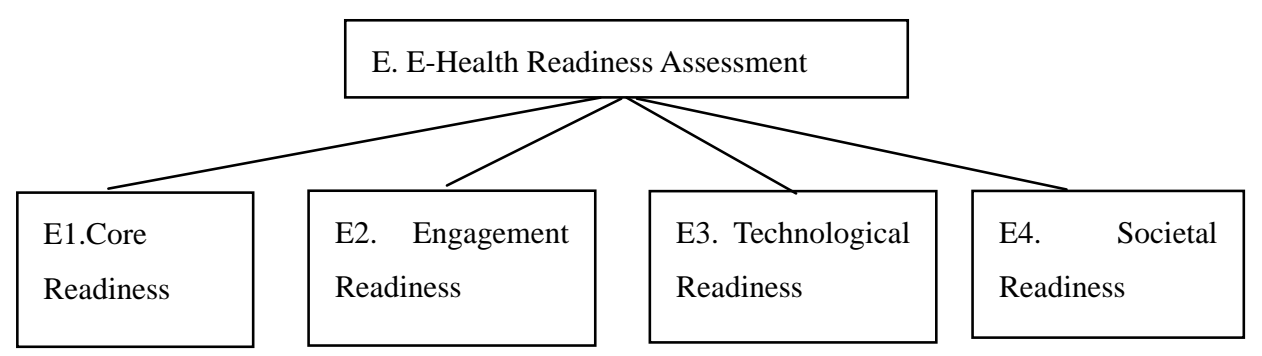

Figure 2. Overall E-Health Readiness Assessment Construct

Each component has its own constructs based on the discussion in Section 4.1.

\section{Core Readiness}

Core readiness assessment result is determined by the variables at the bottom level (See Figure 3), i.e., inefficient documentation of patient records, breached patient privacy, healthcare providers' dissatisfaction with completeness and accuracy, as well as sharing of patient records.

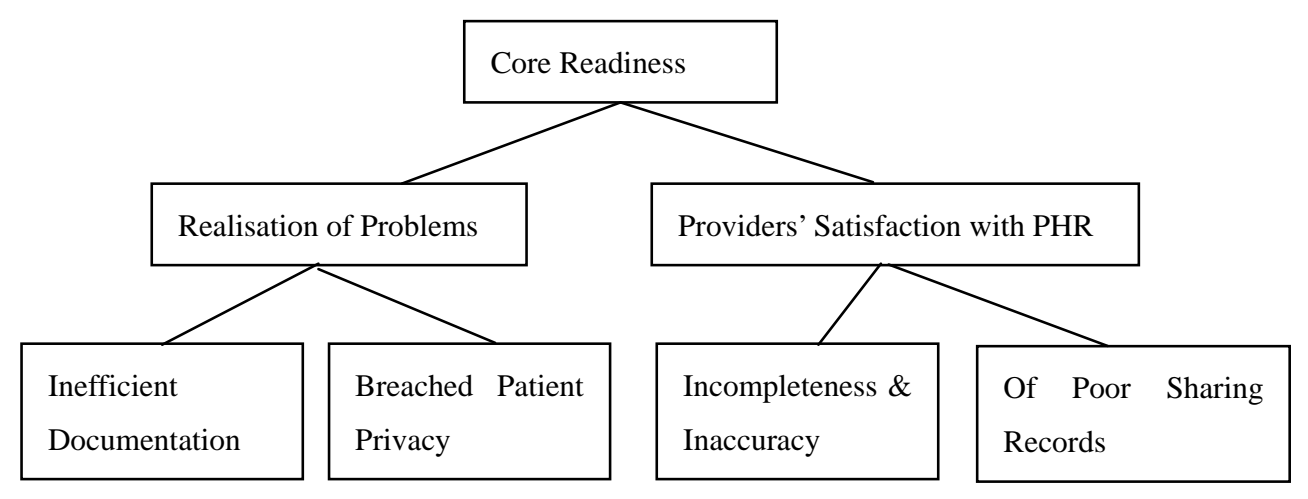

Figure 3. Core Readiness Construct

\section{Engagement Readiness}

Engagement readiness assessment result is dependent on healthcare providers' fear or concern about potentially negative impacts, recognition of benefits of EHR and their willingness to accept EHR training. (Figure 4) 


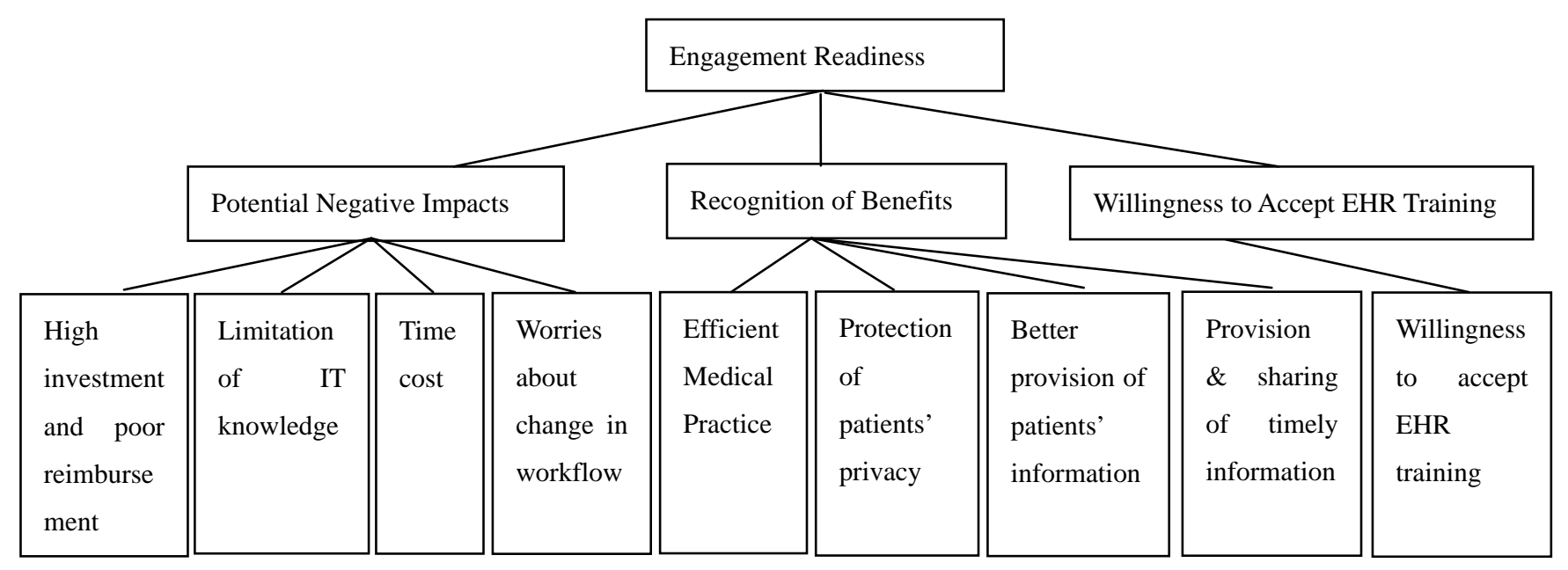

Figure 4. Engagement Readiness Construct

\section{Technological Readiness}

Technological readiness assessment result is dependent on hardware required for EHR applications, network, EHR related software, IT support personnel and healthcare providers' past IT experience. (Figure 5) Regarding each aspect, different indicators are listed in Table 3.

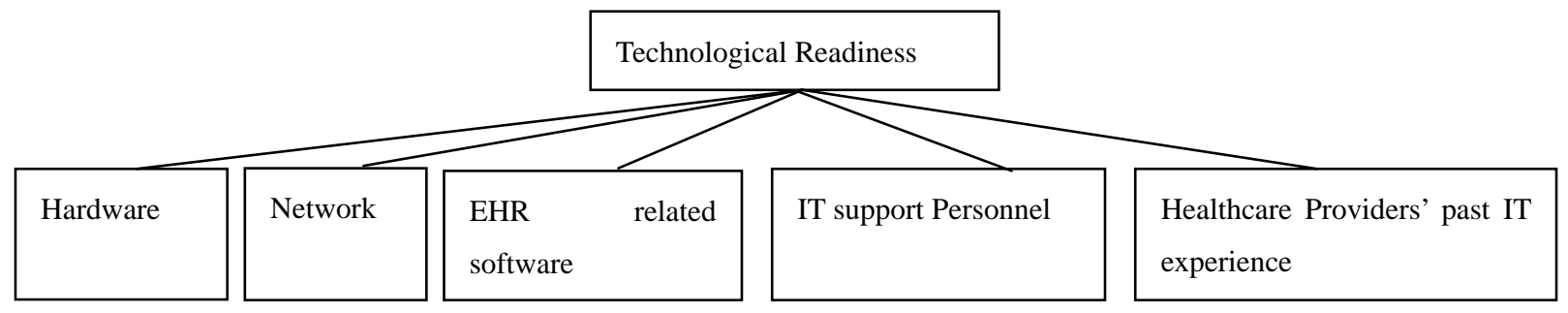

Figure 5. Technological Readiness Construct 


\begin{tabular}{|l|l|}
\hline & Indicators \\
\hline Hardware & 1. Laptop \\
& 2. Desktop \\
& 3. Monitor \\
& 4. Printer \\
& 5. Document scanner \\
& 6. Photocopier \\
& 7. Phone \\
& 8. TV based conferencing \\
& 9. PC based conferencing \\
& 10. Web cam connected \\
& 11. Digitalised X-Ray Equipment \\
& 12. High resolution digital cam monitored on a Microscope \\
\hline Network & 1. Internet access \\
\hline EHR & related \\
software & 2. Maintenance of EHR \\
& 3. To send emails \\
& 4. Standard software \\
\hline IT & 1. Provision of technical support \\
Personnel & 2. Technical support personnel \\
\hline Healthcare & 1. Frequency of using PC \\
Providers' past IT & 2. Frequency of using e-media \\
experience & 3. Computer users for E-Health \\
& 4. Training or direct experience in using EHR \\
\hline
\end{tabular}

Table 3. Indicators for Technological Readiness

\section{Societal Readiness}

Societal readiness assessment result is determined by organisational communication links to both hospitals and administrative centres, provision of care in collaboration with other healthcare organisations, and internal communication frequency and medium used. (Figure 6)

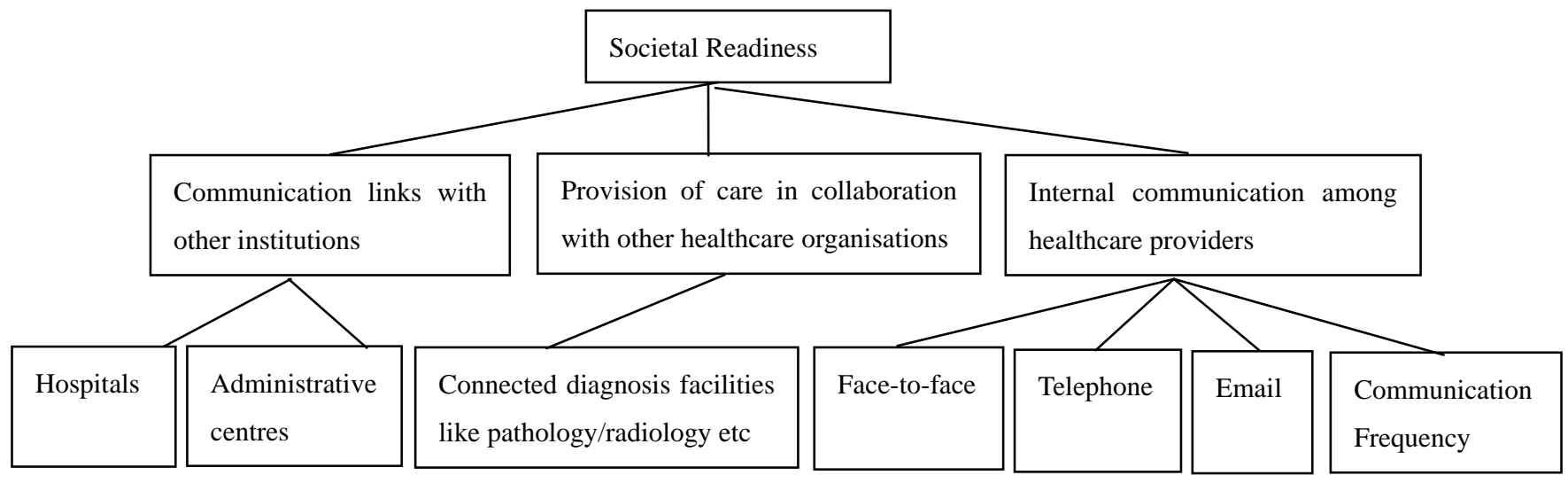

Figure 6. Societal Readiness Construct 


\subsection{Method of Quantifying Constructs Using Connected Graph Theory}

In this step, the constructs and their indicators are expressed using a graph theory (Chattopadhyay et al. 2008). The purpose behind this approach is to quantify the constructs and thus rank overall E-Health readiness level (e.g. as low, low-medium, medium, medium-high or high).

Graph theory essentially provides mathematical structures to model pair wise relations between objects from certain groups. A graph in this context refers to a collection of nodes or vertices as well as collected edges or leafs. A graph structure can be extended by assigning a weight to each edge of the graph. Graphs with weights are called as weighted graphs that are used to represent structures in which pair wise connections have some numerical values. For example if a graph represents the core readiness construct of E-Health readiness assessment, the weights could represent the parameters (i.e. realisation of problems and healthcare providers' satisfaction with PHR) that decide on the core readiness level. This is essentially the motivation behind using graph-based approach in the present work. A digraph with weighted edges in the context of graph theory is called a network that has been used specifically in this paper. Many applications of graph theory exist in the form of network analysis. These split broadly into two categories - 1) analysis to determine structural properties of a network, such as the distribution of vertex degrees and the diameter of the graph, and 2) analysis to find a measurable quantity within the network. Graph theory has been a popular technique for network analysis, such as biological networks related to molecular biology (Aittokallio et al. 2006), physiological network (Stam et al. 2007) and social network related to sociology research (Newman et al. 2002). The present work focuses on the E-Health readiness assessment that is explained based on the four constructs: Core, Engagement, Technological and Societal. It is important to mention here that due the space constraint, only core readiness construct is used to describe the framework. Similar approach can be used to assess other constructs.

It is worth mentioning that there are other related approaches, e.g. hierarchical 
modeling, clustering, and classification-based algorithms that could also be used to measure the readiness level mathematically. As graph theory is inherently more distinctively presentable and moreover the E-Health assessment concept is attempted to be modeled from scratch, authors are more comfortable with graph-based approach at present. However, other approaches as mentioned before could be tested as future studies.

\section{Graph-based model: (Core Readiness)}

Core readiness construct can be explained as a connected graph ' $G$ ' that has two child nodes, 'realisation of problems' and 'providers' satisfaction with PHR computers' (refer to Figure 7). Realisation of problems can be reflected by inefficient documentation and Breached patient privacy, i.e. two sub nodes. Similarly providers’ satisfaction with PHR has two sub nodes (incompleteness and inaccuracy, and of poor sharing records). Each is connected with the parent node. The edges are directed from these sub nodes to their respective parent nodes and each vertex has got a label or name and thus the graph ' $G$ ' may be called as a 'labeled directed graph'. The graph ' $G$ ' therefore can be represented by two sub graphs, such as ' $G 1$ ' and ' $G 2$ ', i.e. G\{G1,G2\}. G1 and G2 consists of vertices $\{R P, I D, B P P\}$ and $\{P S, I I, P S R\}$ respectively. Edges are defined as 'E' $\{\mathrm{E} 1\{\mathrm{E} 1 \mathrm{a}, \mathrm{E} 1 \mathrm{~b}\}, \mathrm{E} 2\{\mathrm{E} 2 \mathrm{a}, \mathrm{E} 2 \mathrm{~b}\}\}$ and having weight ' $w$ ' $\{w 1\{w 1 a, w 1 b\}, w 2\{w 2 a, w 2 b\}\}$. As mentioned, likewise, we may have graphs for the other constructs, i.e. Engagement, Technological and Societal readiness. In this aspect it is important to state that the weights may be generated by survey respondents through questionnaire and their varied opinions could be dealt by calculating the mode, i.e. the most frequent answer on numeric representation.

Vertices can be labeled with natural number that may represent the degree of providers' dissatisfaction with completeness and accuracy of paper-based health records (e.g. 5-point scale). The values can be obtained from questionnaire-based survey data. 


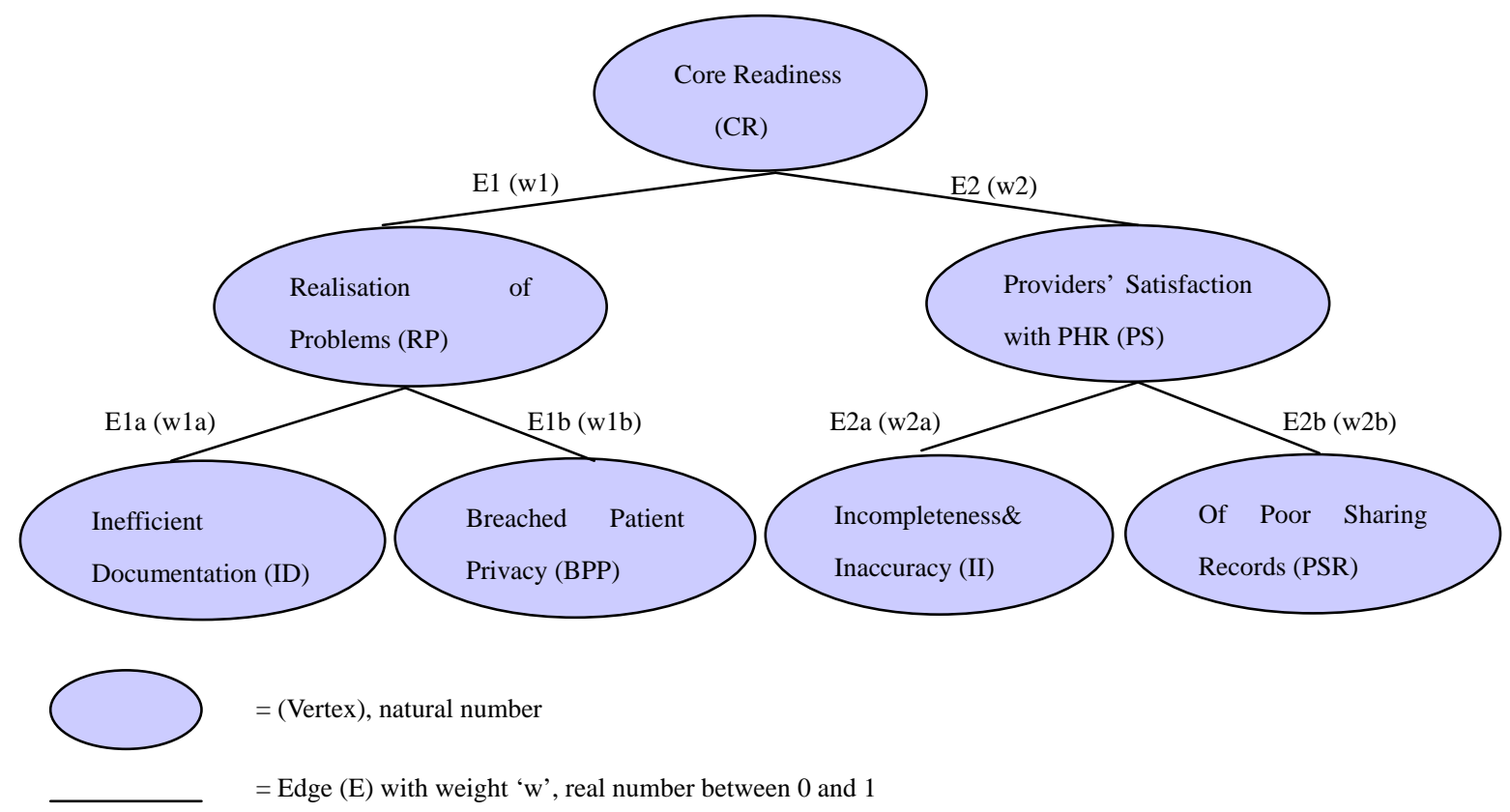

Figure 7. A labeled simple directed weighted graph ' $G$ ' having vertex set $V=\{C R\{R P\{I D, B P P\}, P S\{I$, PSR $\}\}$, Edge set $E=$ $\{\{E 1\{E 1 a, E 1 b\}, E 2\{E 2 a, E 2 b\}$ and weights ' $w$ '

Now the value of the construct 'Core Readiness' can be calculated by the following steps (a bottom-up approach),

$$
\begin{aligned}
& \{(\mathrm{ID} * \mathrm{w} 1 \mathrm{a})+(\mathrm{BPP} * \mathrm{w} 1 \mathrm{~b})\}={ }^{\prime} \mathrm{RP} \\
& \{(\mathrm{II} * \mathrm{w} 2 \mathrm{a})+(\mathrm{PSR} * \mathrm{w} 2 \mathrm{~B})\}=' \mathrm{PS} ' \\
& \{(\mathrm{RP} * \mathrm{w} 1)+(\mathrm{PS} * \mathrm{w} 2)\}={ }^{\prime} \mathrm{CR} '
\end{aligned}
$$

Here, ' $w$ ' are the weights of the respective leaves and $\{\mathrm{CR}\{\mathrm{RP}\{\mathrm{ID}, \mathrm{BPP}\}, \operatorname{PS}\{\mathrm{II}$, PSR \} \} \} are vertices. Similarly, values can be assigned to the other constructs for E-Health readiness assessment and consequently overall E-Health readiness can be revealed using the bottom-up approach. It is important to mention here, this graph-based model is a novel way of looking at the E-Health readiness level. The limitation of it is that, it is a conceptual model and needs validations with real-world data in E-Health scenarios.

\section{CONCLUSIONS}

E-Health systems (e.g. EHR) have been proven to improve healthcare by providing better evidence-based healthcare, increasing medical practice efficiency and the like. 
Nevertheless, their implementation in terms of adoption rate and acceptance still poses a big problem, even in some developed countries. The success of EHR adoption tends to be low for resource constrained developing countries. E-Health readiness assessment, as discussed in Section 2 is an essential requirement for successful E-Health implementation and use. Current E-Health readiness frameworks were criticised for their inconsistent coverage of evaluation components and unclear measures of readiness level. Accordingly, the research contribution of this paper is essentially two-fold. They are

1. E-Health readiness components and indicators from EHR perspective were identified for the development of EHRAF. The components were integrated from the reviewed E-Health readiness frameworks and reflected healthcare providers' and organisational perspectives; and

2. Structuring E-Health readiness constructs and their respective indicators by developing connected graph-based trees and assigning values to each construct with a bottom-up approach. Evaluators can directly use this framework to reveal overall E-Health readiness level for organisations that plan to or will implement E-Health systems.

It is also important to mention that the work has a main limitation. The framework (EHRAF) has not yet been validated with real data from healthcare organisations. Consequently, there is a need for further investigation in this area. The future work involves:

1. Studies are required to validate EHRAF. For example, case studies can be conducted to assess E-Health readiness in healthcare organisations.

2. A survey is required to establish the weights of different leaves in readiness constructs. The survey can be issued as questionnaires or interviews to E-Health experts.

3. EHRAF was developed from EHR perspective and in the future it can be studied from the perspective of holistic E-Health systems (e.g. telemedicine and e-referral systems). The future study is supposed to start with the modification of the 
readiness components but focus on the coverage of the core readiness, as other E-Health systems provide different types of service but use the same platform (e.g. ICT infrastructure, communication links, healthcare providers) that EHR uses.

4. EHRAF was only concerned with healthcare providers' and organisational readiness. A more comprehensive framework needs to incorporate components from patients', system and public perspectives according to future evaluation needs.

\section{REFERENCES}

Agarwal R. and Lucas, H. (2005) The information systems identity crisis:

Focusing on high-visibility and high impact research. MIS Quarterly, 29(3), 381-397.

Aittokallio T., Schwikowski B. (2006) Graph-based methods for analysing networks in cell biology. Brief Bioinform. Vol. 7, No. 3, pp. 243-255.

Alexander H. (2007) Health-service evaluations: Should we expect the results to change practice? Evaluation, 9(4), 405-414.

Allan J. and Englebright J. (2000) Patient-centered documentation: an effective and efficient use of clinical information systems, J Nurs Adm 30, pp. 90-95.

Ammenwerth E., Eichstadter R., Haux R., Pohl U., Rebel S. and Ziegler S. (2001) A randomized evaluation of a computer-based nursing documentation system, Methods Inf Med 40, pp. 61-68.

Ammenwerth E., Brender J., Nykanen P., Prokosch H.U., Rigby M., Talmon J. et al. (2004) Visions and strategies to improve evaluation of health information systems; Reflections and lessons based on the HIS-EVAL workshop in Innsbruck. Int J Med Inf. Jun 30; 73(6):479-91.

Ash J.S. and Bates D.W. (2005) Factors and forces affecting EHR system adoption: report of a 2004 ACMI discussion. J Am Med Inform Assoc; 12:8-12.

Ash J.S., Sittig D.F., Poon E.G., Guappone K., Campbell E. and Dykstra R.H. (2007) The Extent and Importance of Unintended Consequences Related to Computerized Provider Order Entry. J. Am. Med. Inform. Assoc.14(4):415-423. 
Bakker A.R. (2007) The need to know the history of the use of digital patient data, in particular the EHR. International Journal of Medical Informatics, Volume 76, Issues 5-6, May-June, Pages 438-441

Braa, J., Monteiro, E., and Sahay. (2004) Networks of Action: Sustainable health information systems across developing countries. MIS Quarterly, 28(3), 337-362.

Bates D.W. (2005) Physicians and ambulatory electronic health records. Health Affairs (HEALTH AFF), Sep-Oct; 24(5): 1180-9.

BC eHealth Steering Committee. (2005) BC eHealth Conceptual System Architecture. [Internet] Available: http://72.14.253.104/search?q=cache:xTAHDFQOoOsJ:www.health.gov.bc.ca/library/ publications/year/2005/BC_eHealthcas.pdf+\%22ehealth+system $\% 22+$ complex\&hl=e $\underline{\mathrm{n} \& \mathrm{ct}=\mathrm{clnk} \& \mathrm{~cd}=2 \& \mathrm{gl}=\mathrm{au}}$ (Accessed 15 Sep, 2007)

Blobel B. (2004) Authorization and access control for electronic health record systems, Int. J. Med. Inform. 73, pp. 251-257.

Blobel B. (2006) Advanced and secure architectural EHR approaches

International Journal of Medical Informatics, Volume 75, Issues 3-4, March-April, Pages 185-190

Brender J. (1999) Methodology for constructive assessment of IT-based systems-in an organizational context. Int J Med. Inform; 56:67-86.

Brender J. (2006). Evaluation Methods for Health Informatics. Elsevier Inc. London, UK.

Burt C.W. and Hing E. (2005) Use of computerized clinical support systems in medical settings: United States, 2001-03. Medicine: Adv Data; 353:1-5.

Callioni P. (2006) Successful change management. [Internet] Available: www.ehealthexpo.com.au/content/view/59/45/ (Accessed 15 Sep, 2007)

Campbell J.D., Harris K.D. and Hodge R. (2001) Introducing telemedicine technology to rural physicians and settings. J Fam Pract; 50:419-24.

Chattopadhyay S., Li J., Land L. and Ray P. (2008) A Framework for Assessing ICT Preparedness for e-Health Implementation. IEEE 10th International Conference on e-Health Networking, Application \& Services, 7-9 July, Singapore.

Coiera E.and Clarke R. (2004) e-Consent: the design and implementation of consumer 
consent mechanisms in an electronic environment, J. Am. Med. Inform. Assoc. 11 (2), pp. 129-140.

Demiris G., Oliver D.R.P., Porock D. and Courtney K. (2004) Home telehealth: The Missouri telehospice project: Background and next steps. Home Health Care Technology Report; 1:49,55-7.

Dickinson G., Fischetti L. and Heard S. (2004) HL EHR System Functional Model: Draft Standard for Trial Use, July 2004. [Internet] Available: www.hl7.org/ehr/downloads/index_2004.asp. (Accessed $2^{\text {nd }}$, April, 2007).

Diero L., Rotich J.K., Bii J., Mamlin B.W., Einterz R.M., Kalamai I.Z., Tierney W.M. (2006) A computer-based medical record system and personal digital assistants to assess and follow patients with respiratory tract infections visiting a rural Kenyan health centre. BMC Med Inform Decis Mak. Apr 10;6:21.

Embi P.J., Yackel T.R., Logan J.R., Bowen J.L., Cooney T.G. and Gorman P.N. (2004) Impacts of Computerized Physician Documentation in a Teaching Hospital: Perceptions of Faculty and Resident Physicians. J. Am. Med. Inform. Assoc.11(4):300-309.

Eric W. Ford, Menachemi N and Phillips M.T. (2006) Predicting the Adoption of Electronic Health Records by Physicians: When Will Health Care be Paperless? Journal of American Medical Informatics Association: 13(1):106-112.

Eysenbach G. (2001) What is e-health? [editorial]. J Med Internet Res, 1(2), e20.

Field J.M. (1996). Telemedicine: A Guide to Assessing Telecommunications in Health Care. Washington: National Academy Press.

Fullerton C., Aponte P., Hopkins R. III., Bragg D., Ballard D.J. (2006) Lessons learned from pilot site implementation of an ambulatory electronic health record. Baylor University Medical Center Proceedings (BAYLOR UNIV MED CENT PROC), 2006 Oct; 19(4): 303-9.

Gagnon M.P., Scott R.E. (2005) Striving for evidence in e-health evaluation: lessons from health technology assessment Journal Of Telemedicine And Telecare 11: 34-36 Suppl. 2.

Galpottage P.A.B. and Norris A.C. (2005) Patient consent principles and guidelines for e-consent: a New Zealand perspective, Health Inform. J. 11 (1), pp. 5-18.

Gordon D., Geiger G. (1999) Strategic management of an electronic patient record project using the balanced scorecard. J Healthc Inf Manag; 13:113-23. 
Gritzalis D. and Lambrinoudakis C. (2004) A security architecture for interconnecting health information systems, Int. J. Med. Inform. 73, pp. 305-309.

Haglund H. (2002) The significance of welfare services and their electronic applications for the business activities in the future. [Internet] Available: www.etampere.fi (Accessed February 11, 2003)

Halamka J., Aranow M., Ascenzo C., Bates D.W., Berry K., Debor G., Fefferman J., Glaser J., Heinold J., Stanley J., Stone D.L., Sullivan T.E., Tripathi M. and Wilkinson B. (2006) E-Prescribing Collaboration in Massachusetts: Early Experiences from Regional Prescribing Projects. J. Am. Med. Inform. Assoc. 13(3):239-244.

Hassey A. Gerrett D. Wilson A. (2001) A survey of validity and utility of electronic patient records in a general practice. BMJ. 322(7299):1401-5, Jun 9.

Heeks R., (2008) ICT4D 2.0: The Next Phase of Applying ICT for International Development, IEEE Computer, June.

Jennett P., Jackson A., Healy T., Kazanjian A., Woollard R., Haydt S., Bates J. (2002) Alliance for Building Capacity_Framework for Remote and Rural Readiness in Telehealth. Final Report for CANARIE, June 2002. [Internet] Available: www.fp.ucalgary.ca/telehealth/Projects-Canarie-Final\%20Report,\%20Appendix\%20A .htm (Accessed November 4th, 2007)

Jennett P., Jackson A., Healy T., Ho K., Kazanjian A., Woollard R. et al. (2003) A study of a rural community's readiness for telehealth. J Telemed Telecare; 9:259-63.

Jennett P., Yeo M., Pauls M. and Graham J. (2004) Organizational readiness for telemedicine: implications for success and failure. J Telemed Telecare. 9 Suppl 2: S27-30.

Jennett P., Jackson A., Ho K., Healy T., Kazanjian A., Woollard R. et al. (2005) The essence of telehealth readiness in rural communities: an organizational perspective. Telemed J E Health: 11:137-45.

Jennett P.A., Gagnon M.P., Brandstadt H.K. (2005) Preparing for success: Readiness models for rural telehealth. J Postgrad Med. Oct-Dec; 51(4):279-85.

Khoja S., Scott R., Ishaq A. and Mohsin M. (2007) eHealth International Journal. Testing Reliability of eHealth Readiness Assessment Tools For Developing Countries. ehealth international journal, Volume 3(1).

Leung G.M., Yu P.L.H., Wong I.O.L., Johnston J.M. and Tin K.Y.K. (2003) Incentives and Barriers That Influence Clinical Computerization in Hong Kong: A 
Population-based Physician Survey. J. Am. Med. Inform. Assoc.10(2):201-212.

Ministry of Health, Labour and Welfare, Overview of Survey of Medical Institutions in 2002.

[Internet]

Available:

http://wwwdbtk.mhlw.go.jp/toukei/data/160/2002/gaikyou/0004323/g232.html, (accessed September 16, 2007)

Miscione, G. (2007). Telemedicine in the upper amazon: Inetrplay with local health care practices. MIS Quarterly, 31(2), 403-425.

Newman M.E., Watts D.J., Strogatz S.H. (2002) Random graph models of social networks. Proc Natl Acad Sci U S A. Vol. 99, Suppl. 1, pp. 2566-72.

Nykanen P. (2006) E-Health Systems: Their Use and Visions for the Future. Tampere University, Finland. Idea Group Inc.

Obstfelder A., Moen A. (2006) The electronic patient record in community health services--paradoxes and adjustments in clinical work. Studies in Health Technology \& Informatics. 122:626-31.

Overhage J.M., Perkins S., Tierney W.M. and McDonald C.J. (2001) Controlled trial of direct physician order entry: effects on physicians' time utilization in ambulatory primary care internal medicine practices, J Am Med Inform Assoc 8, pp. 361-371.

Overhage JM, Evans L, Marchibroda J. (2005) Communities' readiness for health information exchange: the national landscape in 2004. J Am Med Inform Assoc;12:107-12.

Protti D. (2002) A proposal to use a balanced scorecard to evaluate information for Health: an information strategy for the modern NHS (1998-2005). Computer Biol Med; 32:221-36.

Protti D. (2007) Comparison of information technology in general practice in 10 countries. Healthc Q.10(2):107-16.

Schade C.P., Sullivan F.M., Lusignan S.D. and Madeley J. (2006) e-Prescribing, Efficiency, Quality: Lessons from the Computerization of UK Family Practice. J. Am. Med. Inform. Assoc.13(5):470-475.

Sek A.C., Cheung N.T., Choy K.M., Wong W.N., Tong A.Y., Fung V.H., Fung M., Ho E. (2007) A territory-wide electronic health record--from concept to practicality: the Hong Kong experience. Medinfo. 12(Pt 1):293-6. 
Silber D. (2003). The case for ehealth. European Commission, Information Society, eHealth Conference, Atlanta, Belgium.

Stam C.J., Reijneveld J.C. (2007) Graph theoretical analysis of complex networks in the brain. Nonlinear Biomed Phys. Vol. 1, No. 1, pp. 3.

Staroselsky M., Volk L.A., Tsurikova R., Pizziferri L., Lippincott M., Wald J. and Bates D.W. (2006) Improving electronic health record (EHR) accuracy and increasing compliance with health maintenance clinical guidelines through patient access and input International Journal of Medical Informatics, Volume 75, Issues 10-11, October-November, Pages 693-700.

Steward D.W., Shamdasani P.N. (1990) Focus groups: theory and practice. Newbury Park: Sage Publications. Applied Social Research Methods Series. Vol 20.

Stewart W.F., Shah N.R., Selna M.J., Paulus R.A., walker J.M. (2007) Bridging the inferential gap: the electronic health record and clinical evidence. Health Affairs (HEALTH AFF), Mar-Apr; 26(2): Web Exclusives: w181-91

Taylor H, Leitman R (eds). (2002) European physicians especially in Sweden, Netherlands, and Denmark, lead in use of electronic medical records. Harris Interactive Health Care News. 2(16):1-3.

Terry N.P. (2004) Electronic health records: international, structural and legal perspectives. J Law Med.;12:26-39.

The Alliance for Building Capacity. Framework for rural and remote readiness in telehealth: Project report for CANARIE; 2002. [Internet] Available: www.fp.ucalgary.ca/telehealth/Projects-Canarie-Final\%20Report, \%20June\%202002.htm (Accessed April 28, 2005)

Walsham G., Robey D., Sahay S. (2007) Foreword: Special Issue On Information Systems In Developing Countries. MIS Quarterly Vol. 31 No. 2, pp. 317-326/June

Watson P.J. (2006) Electronic Health Records -Manual for Developing Countries. [Internet] Available: http://64.233.179.104/scholar?hl=zh-CN\&lr=\&q=cache:RlrElvVm-VEJ:www.wpro.w ho.int/NR/rdonlyres/5753F8CF-8A78-4639-BEFC-F0EE9B3CBA0A/0/EHRmanual. pdf+EHR+adoption+developing+countries (Accessed 28 Feb, 2008)

Watts C and Ibegbulam I. (2005). Access to electronic healthcare information resources in developing countries: experiences from the Medical Library, College of Medicine, University of Nigeria. [Internet] Available: http://72.14.253.104/search?q=cache:wT53fzH_TXEJ:www.ifla.org/IV/ifla71/papers/ 164e-Watts_Ibegbulam.pdf+\%22ICT+infrastructure\%22+healthcare+\%22developing 
World Health Organization (WHO). (2005) e-Health Report by Secretariat, $58^{\text {th }}$ World Health Assembly A58/21, April.

WHO. (2006) The global shortage of health workers and its impact. [Internet] Available: www.who.int/mediacentre/factsheets/fs302/en/index.html (Accessed 18 Sep, 2007)

Wickramasinghe N.S., Fadlalla A.M.A., Geisler E., and Schaffer J.L. (2005) A Framework For Assessing E-Health Preparedness. Int. J. Electronic Healthcare, Vol. 1, No. 3. 